\title{
PRODUCCIÓN EXPERIMENTAL DE ROTÍFEROS EN BOLSAS DE PLÁSTICO UTILIZANDO HARINA DE PESCADO COMO FUENTE DE NUTRIENTES
}

\author{
Germán MURRIETA ${ }^{1}$, Jorge NÁJAR ${ }^{2}$, Fernando ALCÁNTARA ${ }^{1}$ \\ 1 Instituto de Investigaciones de la Amazonía Peruana, IIAP. Programa para el Uso y Conservación del Agua y sus Recursos \\ (AQUAREC), Carretera Iquitos - Nauta, Km 4.5. Iquitos, Perú, E-mail : fab_001@hotmail.com \\ 2. Instituto Nacional de Pesquisas da Amazônia, Caixa Postal 478, 69011-970 Manaus, AM, Brazil.
}

\section{RESUMEN}

Este trabajo tuvo como objetivo evaluar la producción de rotíferos en bolsas de plástico, utilizando harina de pescado como fuente de nutrientes. Como unidades experimentales se utilizaron 16 bolsas de plástico de $10 \mathrm{~L}$ de capacidad efectiva. Como fuente de nutrientes se usaron cuatro concentraciones de harina de pescado en cada tratamiento: T1: $0,0 \mathrm{~g} / \mathrm{L}, \mathrm{T} 2: 0,25 \mathrm{~g} / \mathrm{L}, \mathrm{T} 3: 0,50 \mathrm{~g} / \mathrm{L}$ y T4: 0,75 g/L. Las unidades experimentales fueron distribuidas al azar y la duración del experimento fue de once días. El conteo de los rotíferos fue realizado en una cámara de Sedgwick Rafter. Los resultados mostraron que entre el cuarto y el octavo día de experimentación, la producción promedio de organismos incrementó significativamente en proporción al incremento de la oferta de harina de pescado (Promedio \pm Error estándar, T1: $127 \pm 63.5, \mathrm{~T} 2: 277 \pm 138.5$, T3: $356 \pm 178$ y T4: $563 \pm 281.5$ organismos por mililitro; ANOVA, $g l=3, p<0.05)$. El tratamiento cuatro reportó la mayor producción, con1263 organismos por mililitro al onceavo día. Recomendamos utilizar concentraciones altas de harina de pescado para determinar la producción óptima de organismos.

PALABRAS CLAVE: Producción de Rotíferos, harina de pescado.

\section{EXPERIMENTAL PRODUCTION OF ROTIFER IN PLASTIC BAGS USING FISH MEAL AS NUTRIENT SOURCE}

\begin{abstract}
This study aims to evaluate the production of rotifers in plastic bags using fishmeal as nutrient source. Were used 16 plastic bags of $10 \mathrm{~L}$ of effective capacity as experimental units. The concentration of fishmeal was increased in each treatment: T1: $0.0 \mathrm{~g} / \mathrm{L}, \mathrm{T} 2: 0.25 \mathrm{~g} / \mathrm{L} \mathrm{T} 3: 0.50 \mathrm{~g} / \mathrm{L}$ and T4: $0.75 \mathrm{~g} / \mathrm{L}$. The experimental units were randomly distributed and the duration of the experiment was eleven days. Rotifers were counted in a Sedgwick Rafter chamber. The results showed that between the fourth and the eighth day of experimentation, the average production of organisms significantly increased in proportion to the increment of fishmeal supply (Mean \pm SE, T1: $127 \pm 63.5, \mathrm{~T} 2: 277 \pm 138.5, \mathrm{~T} 3: 356 \pm 178$ and T4: $563 \pm 281.5$ organisms per milliliter; ANOVA df $=3 ; \mathrm{p}<0.05$ ). Treatment four have the highest production, with 1263 organisms per milliliter observed at the eleventh day. We recommend using high concentrations of fishmeal to determine the optimum production of organisms.
\end{abstract}

KEYWORDS: Experimental Rotifer production, fish flour 


\section{INTRODUCCIÓN}

En los ecosistemas acuáticos naturales, la supervivencia de las especies depende del equilibrio establecido entre los diferentes niveles de la trama trófica. Así, el desarrollo y supervivencia de larvas y juveniles depende de la presencia de organismos que conforman el fitoplancton y el zooplancton, los cuales se desarrollan en presencia de nutrientes adecuados (FAO, 1989a).

Estudios ejecutados para conocer la composición de las especies de alimento vivo más utilizadas en acuacultura, en diferentes condiciones $\mathrm{y}$ con diferentes tipos de nutrientes, demostraron que el contenido nutricional de estas especies estaba directamente relacionado a su alimento (Fogg, 1975; Watanabe et al., 1978a, 1983; Hirata et al., 1985).

El zooplancton vivo es un buen recurso de proteína, lípidos, minerales, vitaminas y pigmentos carotenoides, destacando para la acuicultura a organismos del Phylum Rotífera (FAO, 1989a).

El rol de los rotíferos en el flujo de energía es de gran importancia, debido a sus cortos ciclos de vida y a las altas tasas reproductivas, implicando una importante conversión de materia orgánica. (Winberg, 1971; Elser et al., 1988). Los rotíferos también participan en el ciclo de los nutrientes y además, en la productividad de los ecosistemas de agua dulce (Makarewicz \& Likens, 1979).

Los rotíferos son los componentes del zooplancton más utilizados en el mundo para alimentar larvas de peces y crustáceos, siendo la base de la cadena alimenticia para el cultivo de otras especies, principalmente por su movimiento en el agua, fácil y económica alimentación con diferentes especies de fitoplancton, ciclo de vida corto y un alto valor nutritivo (Ismiño, 2004; Haché \& Plante, 2011).

La supervivencia larval en los sistemas de cultivo de peces depende, en gran parte, de la disponibilidad de estos organismos. Sin embargo, algunos problemas en su producción masiva, tales como una reducida reproducción y la mortalidad total, se presentan como la principal causa de los mayores impactos económicos en larvicultura, por lo que el comienzo de la alimentación en estadios larvales tempranos parece ser el mayor cuello de botella para el crecimiento industrial del cultivo de peces (Haché \& Plante, 2011).

Este trabajo fue ejecutado con el objetivo de determinar la influencia de la harina de pescado como fertilizante para la producción de rotíferos en cultivo en unidades experimentales compuestas por bolsas de plástico.

\section{MATERIAL Y MÉTODOS}

El trabajo fue realizado en las instalaciones del Instituto de Investigaciones de la Amazonía Peruana (IIAP-Quistococha), entre septiembre y octubre del 2011. Se utilizaron 16 bolsas de plástico de $15 \mathrm{~L}$ como unidades experimentales, siendo sujetadas al techo del cobertizo con alambre galvanizado. (Figuras 1 y 2). En cada bolsa se colocó $10 \mathrm{~L}$ de agua y diferentes cantidades de harina de pescado, pesadas en una balanza analítica (Figura 2), estableciendo cuatro tratamientos con tres réplicas cada uno, como se indica a continuación:

T1: $0,00 \mathrm{gL}^{-1}$ harina pescado

T2: $0,25 \mathrm{gL}^{-1}$ harina pescado

T3: $0,50 \mathrm{gL}^{-1}$ harina pescado

T4: $0,75 \mathrm{gL}^{-1}$ harina pescado

La distribución de los tratamientos fue completamente al azar y determinada, utilizando el programa Random Sequence Generator, (www.random.org/sequences).

Las evaluaciones de las variables físico químicas de los ambientes de cultivo fueron realizadas desde el día siguiente a la instalación del experimento, utilizando para ello un equipo multiparámetros, un oxímetro y un Kit de Aguas Lamotte.

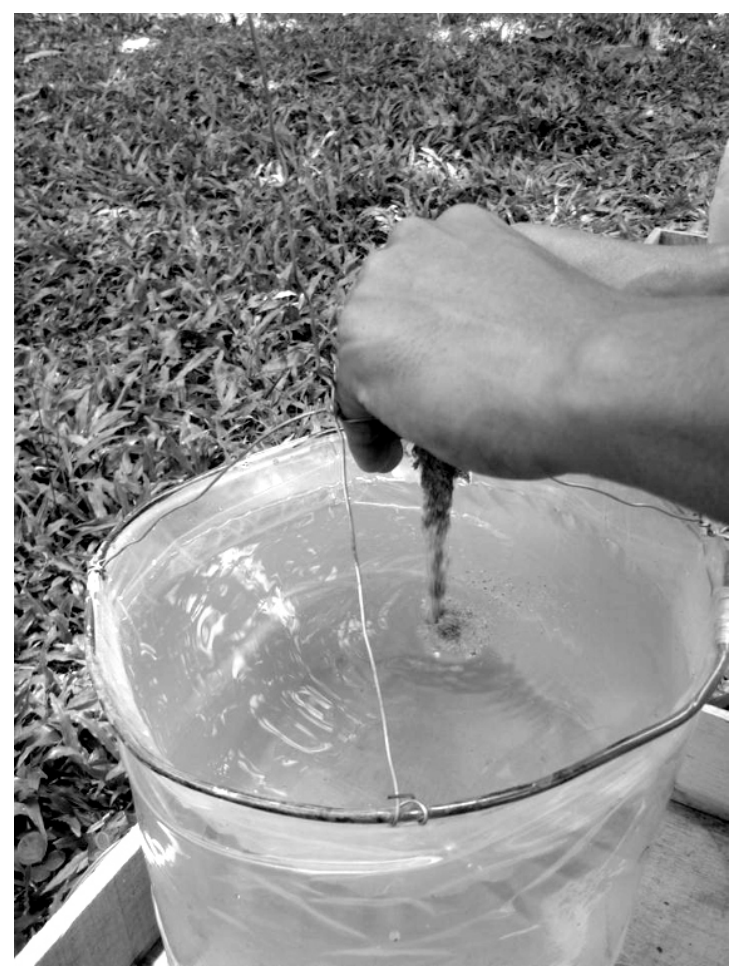

Figura 1. Unidad experimental. 


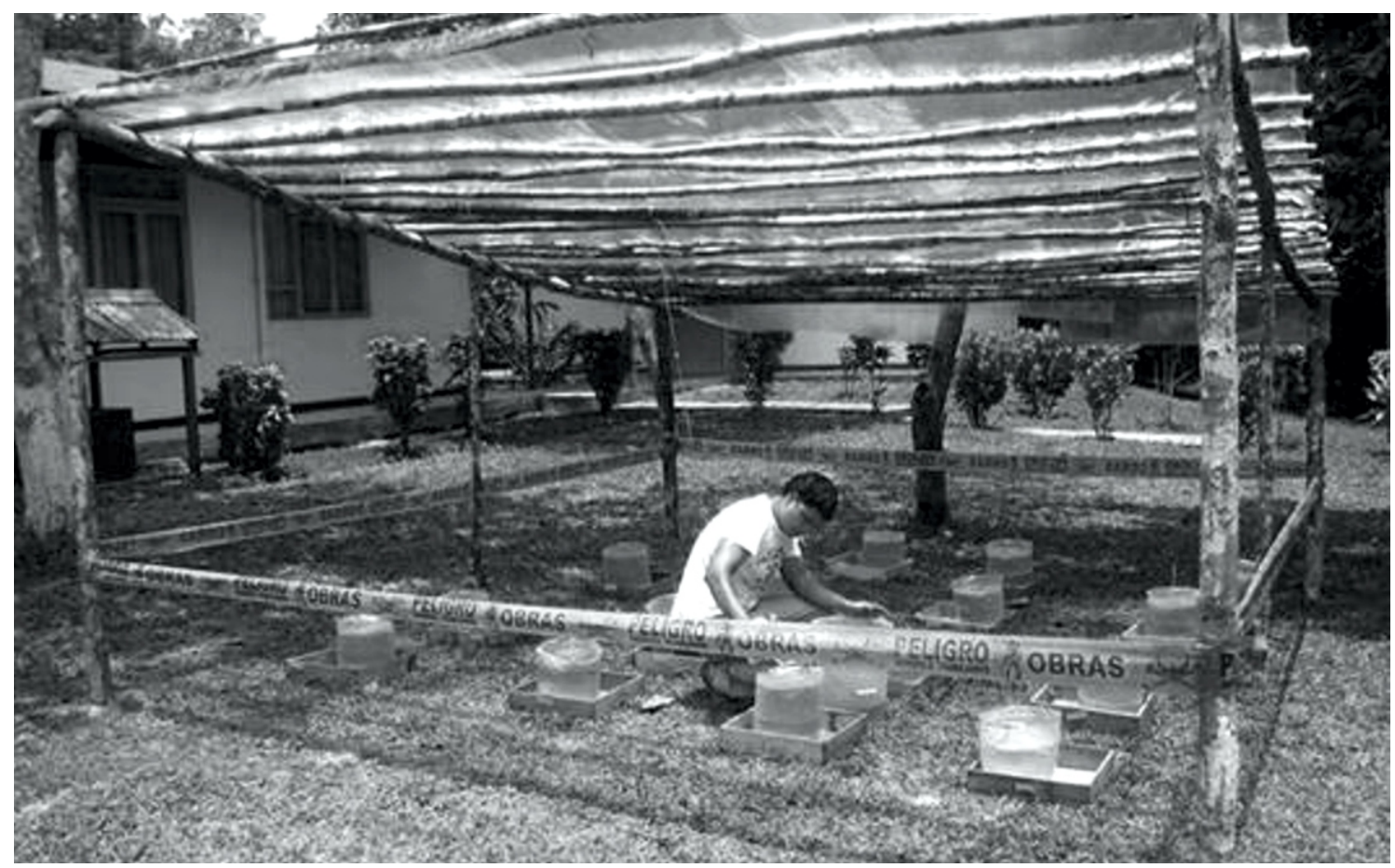

Figura 2. Área experimental para la producción de rotíferos.

La producción de rotíferos fue evaluada diariamente colectando muestras de $1 \mathrm{ml}$ de cada unidad experimental para la cuantificación de rotíferos en el laboratorio, utilizando una cámara Sedgwick Rafter y contando el número de rotíferos de 20 cuadrantes al azar, para luego determinar el promedio de organismos por unidad experimental.

Para determinar las diferencias significativas entre los tratamientos se aplicó un Análisis de Varianza (ANOVA), seguido de la prueba de Tukey $(p<0,05)$. Los análisis se realizaron con el programa Bioestat 5.0.

\section{RESULTADOS Y DISCUSIÓN}

Las variables físico químicas del agua en las unidades de cultivo de los rotíferos se muestran en la tabla 4. Estas variables en promedio permanecieron en niveles adecuados para el desarrollo de los organismos planctónicos, en general y en particular, de los rotíferos. Hagiwara et al. (2007) refieren que la temperatura de cultivo influye en la producción de rotíferos, siendo necesario mantener una temperatura óptima entre 27 a $28{ }^{\circ} \mathrm{C}$. En el presente estudio la temperatura de los diferentes tratamientos osciló en promedio entre los 28 y $29^{\circ} \mathrm{C}$.
Vasileiadou et al. (2009) mencionan que rotíferos del género Brachionus difieren en sus preferencias por temperatura, salinidad, $\mathrm{pH}$ y oxígeno disuelto. En el presente estudio no hubo diferencias entre la temperatura y el $\mathrm{pH}$ de los diferentes tratamientos, pero se observó que el oxígeno disuelto en el tratamiento uno fue sustancialmente menor que en los demás tratamientos, debido a una menor actividad fotosintética derivada de la menor producción de organismos componentes del fitoplancton que dependen de la disponibilidad de nutrientes.

Durante el experimento fue notable el incremento progresivo de los rotíferos a partir del cuarto día en todos los tratamientos, registrando al octavo día el mayor número de organismos en todos los tratamientos, siendo la producción directamente proporcional a la oferta de harina de pescado; es decir, la producción varió como se indica a continuación en los promedios obtenidos para cada tratamiento: T1: 127, T2: 277, T3: 356 y T4: 563 rotíferos por mililitro, estadísticamente diferentes $(\mathrm{p}<0.05)$, observando la mayor producción de 1.263 rotíferos. $\mathrm{mL}^{-1}$ en el tratamiento cuatro al octavo día. Tablas 1 y 2 . Figura 3 . Se observaron diferencias estadísticamente significativas (ANOVA $p<0,05$ ) únicamente entre T1 y T4. Tabla 3. Figura 4 
A partir del noveno día la producción disminuyó considerablemente en los tratamientos tres y cuatro, aumentando ligeramente en los tratamientos uno y dos. Al onceavo día, sin embargo, todos los tratamientos evidenciaron disminución en la producción de rotíferos. Pensamos que esto se debe a la disminución de nutrientes derivados de la harina de pescado, por lo cual, en la posibilidad de producción a mayor escala, se debería considerar como alternativas de acción, la oferta de una dosis adicional de harina de pescado al noveno día de cultivo o el inicio de una nueva etapa de producción.

Ascón en el 1992, registró una producción de 7.800 rotíferos/ml, utilizando estanques de tierra de cuatro metros cuadrados y medio metro de profundidad, fertilizando el agua con $1.5 \mathrm{~kg}$ de gallinaza. $\mathrm{m}^{-2}$ y $30 \mathrm{~g}$ de Superfosfato. $\mathrm{m}^{-2}$. Esta producción es, evidentemente, mucho mayor que la alcanzada en nuestro experimento, pero difícil de replicar por el aporte indeterminado de nutrientes de la gallinaza, compuesta por restos de alimento y excretas de gallina en proporción variable, además del aporte de nutrientes del suelo de las unidades experimentales, sumados a la influencia de la precipitación pluvial que puede diluir los nutrientes y renovar el agua afectando la producción de los organismos en cultivo.

El género de rotífero más cultivado en el mundo es Brachionus, destacándose la especie Brachionus plicatilis, seguida por B. callyciflorus, $B$. rubens, $B$. urceolari y $B$. falcatus. Estas especies están siendo utilizadas con diversos medios enriquecedores con fines de mejorar el rendimiento productivo en la fase de larvicultura de muchas especies de peces neotropicales. Entre los medios destinados a mejorar el efecto nutricional de los Brachionus están la vitamina $\mathrm{C}$, los ácidos grasos superiores eicosapentanoico (EPA) y docosahexanoico (DHA) y los probióticos, entre otros (Hagiwara et al., 2001 citado por Prieto \& Atencio, 2008). La literatura reporta que Brachionus mulleri fue producido utilizando torta de Basia latifolia que contenía urea, superfosfato, sulfato de amonio y harina de huesos, inoculando 20 unidades. $\mathrm{L}^{-1}$ Como resultado obtuvieron una producción de 8.000 y 7.500 organismos/L en tres semanas (Nandy et al., 1976 citado en Ismiño,2004). Brachionus rubens fue cultivado utilizando como alimento Scenedesmus y como fertilizante el estiércol de cerdo y alimento suplementario, alcanzando una densidad de 500 organismos. $\mathrm{mL}^{-1}$ (Groeneweg \& Schluter, 1981 citado en Ismiño, 2004). Por su parte, Ismiño (2004) utilizó harina de pescado para la producción masiva de Scenedesmus y rotíferos, alcanzando una densidad zooplanctónica de 5.592 organismos. $\mathrm{L}^{-1}$ en dieciséis días.

Cisneros 2011, ejecutó el cultivo del rotífero nativo Brachionus sp., cepa Chilca, Perú en recipientes de acrílico de $180 \mathrm{~L}$ a una salinidad de 35 ups y una temperatura constante de $27^{\circ} \mathrm{C}$ en un fotoperiodo de 8:16 L, alimentando el cultivo con oferta de la micro alga Nannochloris maculata y Nannochloropsis oculata, con diversos enriquecedores comerciales como Marol-E (T1), Protein Hufa (DHA $5 \%$, EPA $3.5 \%$, proteínas $42 \%$ ) (T2), Selcon (T3) e Easy Super Selco (T4) reportando la mayor producción de rotíferos de 149 \pm 0.58 indiv. $\mathrm{mL}^{-1}$ con el enriquecedor Protein Hufa. Sobre el uso de los enriquecedores consideramos que, si bien, técnicamente satisfacen la demanda de nutrientes, tienen la dificultad implícita del costo y de su limitada accesibilidad.

En este estudio se utilizó un método sencillo, de bajo costo y accesible tanto física como técnicamente, con buenos resultados, finalizando en una producción de 1.263 rotíferos. $\mathrm{mL}^{-1}$, que atribuimos a la alta disponibilidad de nutrientes presentes en la harina de pescado en niveles suficientes para cubrir las necesidades de las micro algas de soporte de los rotíferos, en adición al suministro directo de micro partículas de harina de pescado para el consumo de los rotíferos.

Tabla 1. Producción de rotíferos utilizando harina de pescado en diferentes niveles (Org.mL $\left.{ }^{-1}\right)$

\begin{tabular}{ccccccc}
\hline Tratamiento & Media & N & Desv. típ. & Mínimo & Máximo & $\begin{array}{c}\text { Error } \\
\text { estándar }\end{array}$ \\
\hline T1 & 127 & 8 & 205 & 0 & 600 & 63.5 \\
T2 & 277 & 8 & 242 & 0 & 587 & 138.5 \\
T3 & 356 & 8 & 224 & 125 & 662 & 178 \\
T4 & 563 & 8 & 334 & 225 & 1263 & 281.5 \\
\hline Total & 330 & 32 & 291 & 0 & 1263 & \\
\hline
\end{tabular}


Tabla 2. ANOVA de la producción de rotíferos utilizando harina de pescado en diferentes niveles (Org.mL $\left.{ }^{-1}\right)$

\begin{tabular}{|c|c|c|c|c|c|c|c|}
\hline & & & $\begin{array}{l}\text { Suma de } \\
\text { cuadrados }\end{array}$ & gl & $\begin{array}{c}\text { Media } \\
\text { cuadrática }\end{array}$ & $\mathbf{F}$ & Sig. \\
\hline Producción & Inter-grupos & (Combinadas) & 791894.531 & 3 & 263964.844 & 4.020 & 0.017 \\
\hline \multicolumn{8}{|l|}{$\left(\text { Org.mL } L^{-1}\right)^{*}$} \\
\hline Tratamiento & Intra-grupos & & 1838398.438 & 28 & 65657.087 & & \\
\hline \multicolumn{3}{|c|}{ Total } & 2630292.969 & 31 & & & \\
\hline
\end{tabular}

Tabla 3. Prueba de Tukey de la producción de rotíferos utilizando harina de pescado en diferentes niveles (Org./ml)

\begin{tabular}{cccc}
\hline Prueba de Tukey & Diferencia & $\mathbf{0}$ & (p) \\
\hline Tratamiento (1 a 2) & 150,3750 & 1,6595 & ns \\
\hline Tratamiento (1 a 3) & 229,8750 & 2,5369 & ns \\
\hline Tratamiento (1 a 4) & 436,1250 & 4,813 & $<0,05$ \\
\hline Tratamiento (2 a 3) & 79,5000 & 0,8773 & ns \\
\hline Tratamiento (2 a 4) & 285,7500 & 3,1535 & ns \\
\hline Tratamiento (3 a 4) & 206,2500 & 2,2761 & ns \\
\hline
\end{tabular}

Tabla 4. Parámetros físico-químicos registrados durante el experimento

\begin{tabular}{cccc}
\hline Tratamiento & Temperatura $\left({ }^{\circ} \mathbf{C}\right)$ & $\mathbf{p H}$ & $\mathbf{0}_{2}\left(\mathbf{m g} \cdot \mathrm{L}^{-1}\right)$ \\
\hline T1 & $28,52 \pm 1,78$ & $9,17 \pm 0,93$ & $5,94 \pm 0,70$ \\
T2 & $28,98 \pm 2,02$ & $10,10 \pm 0,50$ & $7,89 \pm 4,43$ \\
T3 & $29,22 \pm 2,17$ & $9,85 \pm 0,64$ & $7,63 \pm 6,55$ \\
T4 & $29,52 \pm 2,41$ & $9,49 \pm 0,96$ & $6,58 \pm 7,15$ \\
\hline
\end{tabular}




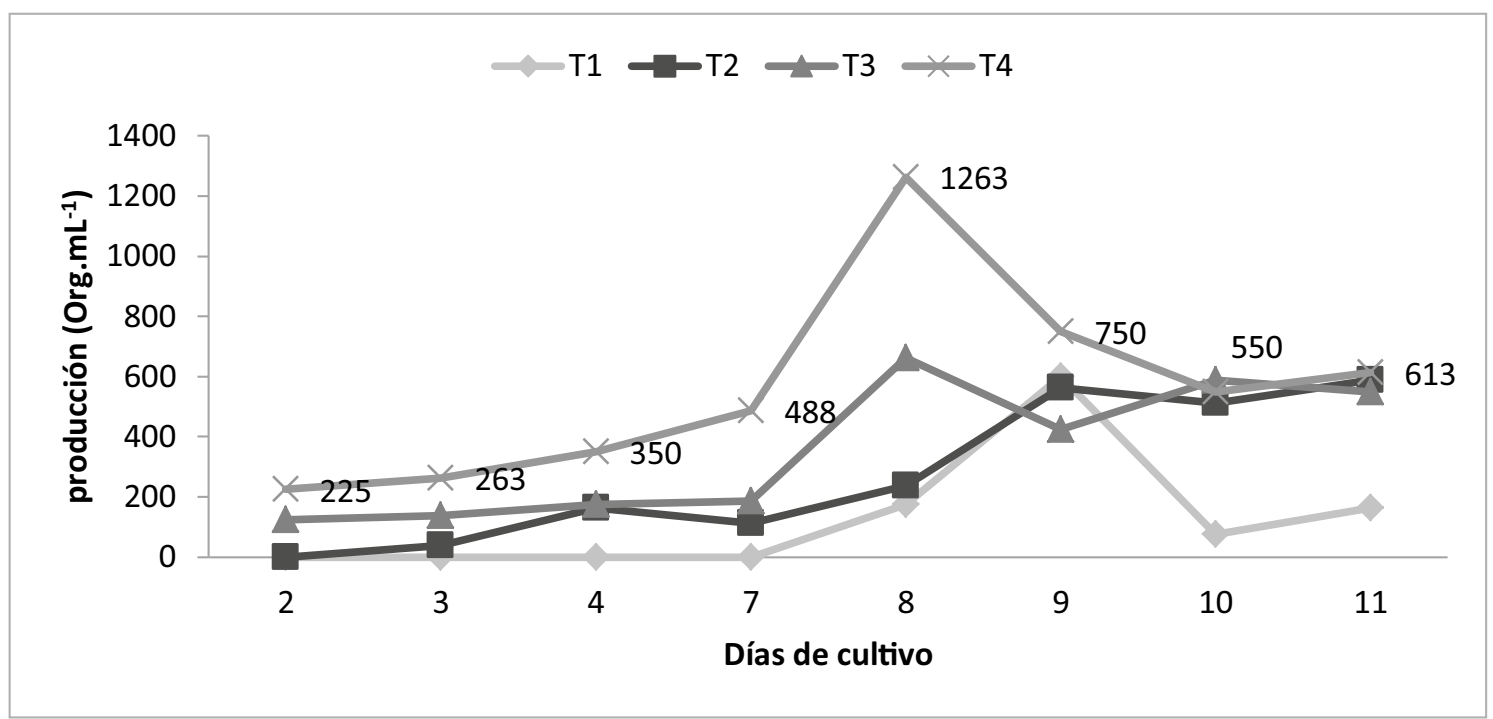

Figura 3. Número de Rotíferos. $\mathrm{mL}^{-1}$ registrados durante el experimento.

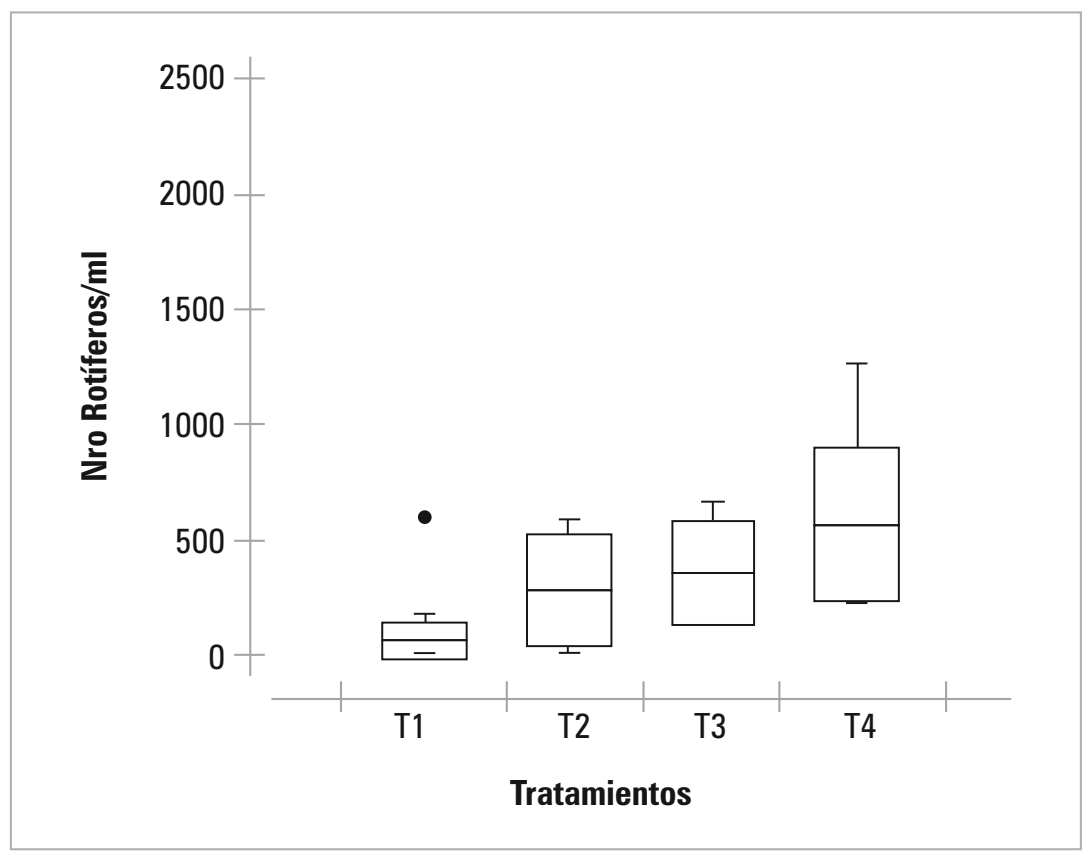

Figura 4. Número de rotíferos. $\mathrm{mL}^{-1}$ registrados durante el experimento, utilizando diferentes niveles de harina de pescado. 


\section{CONCLUSIONES Y RECOMENDACIONES}

La harina de pescado utilizada como fuente de nutrientes en este trabajo dio buenos resultados y resultó eficaz para la producción masiva de rotíferos. El tratamiento cuatro, consistente en la oferta de 0,75 $\mathrm{g}$ de harina de pescado/L de agua, dio los mejores resultados de producción de rotíferos. La cosecha de los rotíferos se puede realizar exitosamente a partir del octavo día de iniciado el cultivo.

Recomendamos utilizar concentraciones mayores de harina de pescado para establecer la cantidad óptima que permita la mayor producción en el menor tiempo posible, aplicando estos criterios simples de producción de rotíferos a escalas de producción masiva.

\section{REFERENCIAS BIBLIOGRÁFICAS}

Ascón, G. D., 1992. Cultivo masivo de rotíferos en estanques de tierra. Folia Amazónica, 4(1): 101106

Cisneros, R. 2011. Rendimiento poblacional del rotífero nativo Brachionus sp. "Cayman", utilizando diferentes enriquecedores. Departamento Académico de Biología, Universidad Nacional Agraria La Molina, Lima - Perú. Ecología Aplicada, 10(2):99-105.

Elser, J. J., Elser, M. M., Mackay, N. A. \& Carpenter, S. R., 1988, Zooplankton-mediated transitions between $\mathrm{N}$ - and P-limited algal growth. Limnology and Oceanography, 33(1): 1-14.

FAO, (1989a). La producción de alimento vivo y su importancia en la acuacultura. Documento de campo Nro 12.

Fogg A.E., 1975. Algal cultures and phytoplankton ecology.Second Ed.The University of Wisconsin Press, $175 \mathrm{pp}$.

Haché R. \& Plante S. 2011. The relationship between enrichment, fatty acid profiles and bacterial load in cultured rotifers (Brachionus plicatilis L-strain) and Artemia (Artemia salina strain Franciscana). Aquaculture 311 (2011) 201-208.
Hagiwara A., Suga K., Akazawa A., Kotani T. \& Sakakura Y. 2007. Development of rotifer strains with useful traits for rearing fish larvae. Aquaculture 268: 44-52.

Hirata H., A. Mavrikos\& Y. Shigehisa, 1985. Evaluation of the use of Brachionus plicatilis and Artemia nauplii for rearing prawn Penaeu sjaponicous larvae on a laboratory scale. Mem. Fac. Fish., Kagoshima Univ. 34, No. 1:27-36.

Ismiño, R. 2004. Producción de alimento vivo (Plancton a partir de Harina de pescado). Informe técnico Instituto de Investigaciones de la Amazonía Peruana. 45pp.

Makarewicz, J. C. \& Likens, G. E., 1979, Structure and function of the zooplankton community of Mirror Lake, New Hampshire. Ecological Monographs, 49(1): 109-127.

Prieto, M. Atencio, V. 2008. Zooplancton en la larvicultura de peces neotropicales. rev.mvz córdoba 13(2):1415-1425

Watanabe T., 1978. Nutritive value of plankton for fish larvae in the view point of lipids. Fish.Sarv 22, pp. 93-111. Koseisha-Koseikaku (Tokyo).

Watanabe T., C. Kitajima\& S. Fujita, 1983. Nutritional values of live organisms used in Japan for mass propagation of fish: a review. Aquaculture, 34(1983):115-145.

Winberg, G. G., 1971, Methods for estimating the production of populations with continuous reproduction. In: G. G. Winberg, Methods for the estimation of production of aquatic animals. Translated from the Russian by A. Duncan.Royal Holloway College, University of London, London.

Recibido: 4 de agosto del 2015

Aceptado para publicación: 7 de setiembre del 2015 
